\title{
Potential benefits of L. acidophilus in dyslipidemic rats
}

\author{
Onrapak Reamtong ${ }^{1}$, Tipparat Thiangtrongjit ${ }^{1}$, Nathamon Kosoltanapiwat ${ }^{2}$, \\ Watanalai Panbangred ${ }^{3}$ \& Pattaneeya Prangthip ${ }^{4 凶}$
}

Several studies have shown that probiotics and synbiotics ameliorate dyslipidemia. However, the molecular mechanisms mediating their effects remain to be determined. Therefore, we aimed to compare the effects of a probiotic, a prebiotic, and a synbiotic in dyslipidemic Sprague-Dawley rats, and explore the mechanisms involved using a proteomic approach. The rats were allocated to five groups: a control group that was fed normal chow, and four high-fat diet-fed groups, three of which were administered a probiotic (Lactobacillus acidophilus), a prebiotic (inulin), or a combination of the two (a synbiotic) for 30 days. We showed that the administration of inulin, and especially L. acidophilus, improved the lipid profile and reduced the serum concentrations of inflammatory markers in high-fat diet-fed rats. Proteomic analysis showed changes in lipid elongation, glycerolipid metabolism, activation of antioxidants, and a reduction in the activation of the mitogen-activated protein kinase signaling pathway in the livers of rats administered $L$. acidophilus, which likely mediate its beneficial effects on inflammation and dyslipidemia by reduced the levels of $18.56 \%$ CRP, $35.71 \%$ TNF- $\alpha 25.6 \%$ LDL-C and 28.57\% LDL-C/HDL-C ratio when compared to HF group. L. acidophilus and inulin may represent effective natural means of maintaining inflammation and dyslipidemia.

Overweight and obesity are major public health problems worldwide, and their prevalences have continuously increased during recent decades. In 2016, approximately $13 \%$ of adults were obese worldwide ${ }^{1}$, but increases in the prevalence of overweight and obesity have also occurred in children and adolescents. Overweight and obesity increase the risks of heart disease and related mortality, through the associated dyslipidemia and low high-density lipoprotein (HDL)-cholesterol concentration ${ }^{2}$. Dyslipidemia was reported to relate to the activation of inflammation at least by increasing the production of inflammatory cytokines such as tumor necrosis factor alpha and interleukin 6. In reverse, cytokines could disrupt lipid metabolism, which has crucial roles in the pathogenesis of atherosclerosis ${ }^{3}$. The consumption of energy-dense foods that contain high proportions of fats and sugars is the principal cause of overweight and obesity ${ }^{4}$. This can be modeled in rats by feeding them a high-fat diet (e.g., $22.59 \mathrm{~kJ} / \mathrm{g}$ from fat), which induces dyslipidemia and defects in hepatic fat metabolism ${ }^{5,6}$.

Probiotics are live microorganisms that have health benefits for their hosts ${ }^{7}$. More than 500 different microbial species have been found in the human intestinal tract, and among these is Lactobacillus acidophilus, which is a well-known species that is commonly found in yogurt. It usually colonizes the large intestine of newborns and remains in the intestinal tract of humans until death ${ }^{8}$. L. acidophilus has been shown to be associated with greater longevity and to have a hypocholesterolemic effect ${ }^{9}$; for example, it lowers serum total cholesterol, special on low density lipoprotein cholesterol in pigs ${ }^{10}$. Prebiotics are indigestible food components that selectively stimulate the growth and activity of probiotic species in the colon, and also reduce serum total cholesterol (TC), low-density lipoprotein (LDL)-cholesterol, and triglyceride (TG) concentrations ${ }^{11}$. Inulin is an indigestible polysaccharide that is produced by plants, which was approved for use as a prebiotic in the United States by the Food and Drug Administration in 2018, because it improves the nutritional value of manufactured food products ${ }^{12}$. Specifically, inulin has been reported to reduce body weight gain, liver weight, and the serum and hepatic TC and TG concentrations in mice ${ }^{13}$. Furthermore, the consumption of a combination of prebiotics and probiotics, termed a synbiotic, has been reported to improve host health ${ }^{14}$. For example, the synbiotic mixture of Lactobacillus plantarum S58 and hull-less barley $\beta$-glucan has been shown to reduce lipid accumulation in high-fat diet-fed mice $^{15}$. However, the effects of a synbiotic composed of L. acidophilus and inulin on lipid metabolism have not been investigated in an animal model, and the molecular mechanisms involved remain to be determined.

\footnotetext{
${ }^{1}$ Department of Molecular Tropical Medicine and Genetics, Faculty of Tropical Medicine, Mahidol University, Bangkok, Thailand. ${ }^{2}$ Department of Microbiology and Immunology, Faculty of Tropical Medicine, Mahidol University, Bangkok, Thailand. ${ }^{3}$ Department of Biotechnology, Faculty of Science, Mahidol University, Bangkok, Thailand. "'Department of Tropical Nutrition and Food Science, Faculty of Tropical Medicine, Mahidol University, 420/6 Ratchawithi Road, Ratchathewi, Bangkok 10400, Thailand. ${ }^{\circledR}$ email: pattaneeya.pra@mahidol.ac.th
} 
Body weight (g), food consumption (KJ/day) and liver weight (g) for 4 weeks

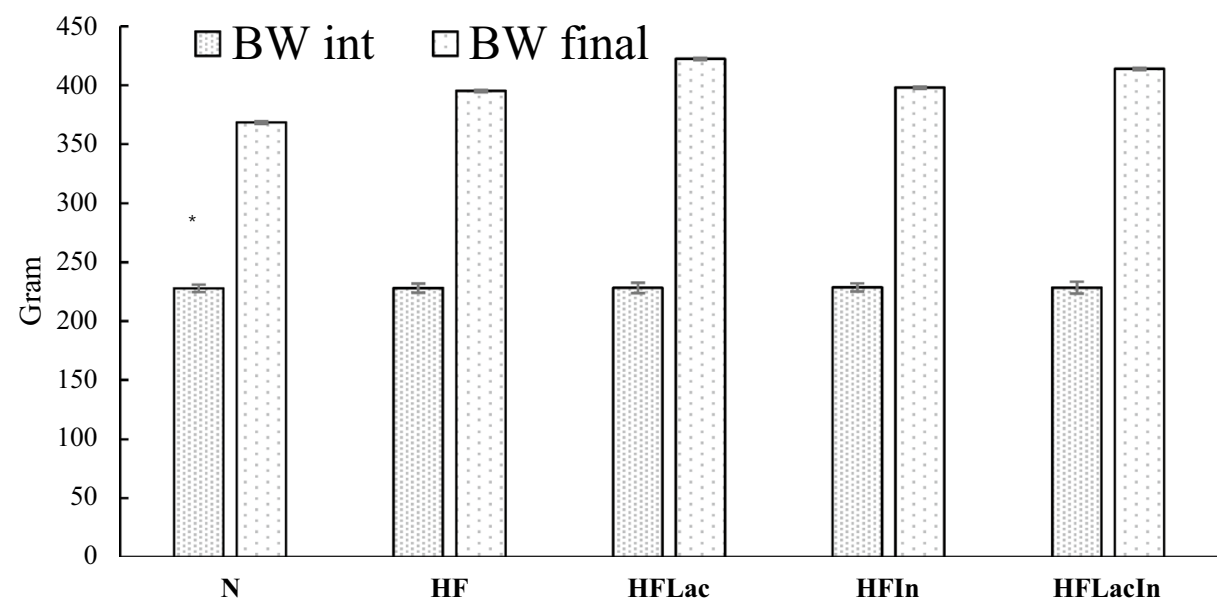

\begin{tabular}{lcccccc}
\hline & $\mathrm{N}$ & $\mathrm{HF}$ & \multicolumn{1}{c}{ HFLac } & HFIn & HFLacIn \\
\hline Food Consumed $(\mathrm{KJ})$ & $740 \pm 3.0$ & $966 \pm 11.2$ & $882 \pm 3.1$ & $861 \pm 13.4$ & $978 \pm 11.4$ \\
Liver (gram) & $12 \pm 0.57$ & $15 \pm 0.48$ & $16 \pm 0.42$ & $14 \pm 0.69$ & $15 \pm 0.35$
\end{tabular}

Figure 1. Body mass (g), food consumption (KJ/day), and liver mass ( $g$ ) of the rats in each group $(n=13)$. (N) Rat fed a normal diet (HF) Rats fed with a high fat diet (HFLac, HFIn, HFLacIn,) Rats fed with a high fat diet and L. acidophilus, inulin, and L. acidophilus + inulin, respectively.

In the present study, we studied five groups of rats: one fed a normal diet $(\mathrm{N})$, and four fed a high-fat diet (HF), three of which were also administered L. acidophilus (HFLac), inulin (HFIn), or both L. acidophilus and inulin (HFLacIn). After 30 days, blood was collected for biochemical analyses and to measure inflammatory marker concentrations. The rats were euthanized and their livers were weighed and subjected to histological and proteomic analysis. In this way we have characterized the host lipid profile associated with L. acidophilus and inulin consumption, and identified key proteins that are involved in the mechanisms of the effects of this synbiotic combination.

\section{Results}

Rat body and liver masses. The body and liver masses were measured for all the groups (Fig. 1). Highfat diet-feeding for 4 weeks caused greater weight gain than the consumption of a normal diet. The HFLac and HFLacIn groups consumed $882 \pm 3.1$ and $978 \pm 11.4 \mathrm{~kJ} /$ day, which represented larger energy intakes than in the other groups. However, the administration of L. acidophilus, prebiotic, or synbiotic for 4 weeks did not have significant effects on the body mass of high-fat diet-fed rats. The liver masses of all the HF groups were higher than that of the normal group. However, L. acidophilus, prebiotic, and synbiotic administration for 4 weeks did not significantly affect this parameter.

Serum lipid profile. Serum lipid concentrations were measured in all the groups of rats (Fig. 2). The N group demonstrated the highest HDL-C concentration, and the concentrations were significantly lower in all the HF groups. HFLac and HFLacIn group reduced the LDL-C concentration by $25.6 \%$ and $17 \%$ respectively, and this effect was not pronounced in the HFIn group (Fig. 2A). Calculation of the LDL-C/HDL-C ratio (Fig. 2B) demonstrated that the HFLac group had the most advantageous lipid profile of the HF groups by reduction of the $28.57 \%$ of LDL-C/HDL-C ratio.

Liver and kidney-related parameters. Serum liver and kidney-related parameters were measured in all the groups (Fig. 3). The N group had the lowest AST, ALT, and ALP activities, and high-fat diet-feeding increased the activities of all these enzymes, which reflect recent liver damage, not residual function. The administration of a probiotic, prebiotic, or synbiotic combination for 4 weeks did not significantly affect the AST, ALT, or ALP activities. Kidney function was assessed by the measurement of BUN and creatinine in each group, and the values were in the ranges $11.5-13.1 \mathrm{mg} / \mathrm{dl}$ and $\sim 0.2 \mathrm{mg} / \mathrm{dl}$, respectively. No statistically significant differences were found among the groups.

Serum inflammatory marker concentrations. TNF- $\alpha$ is a cytokine that plays an important role in inflammation, such that a high serum concentration of TNF- $\alpha$ reflects systemic inflammation. We measured the serum TNF- $\alpha$ concentration of rats in all the groups by ELISA, and found that the serum TNF- $\alpha$ was higher 
High Density Lipoprotein; HDL and Low Density

Lipoprotein; LDL Cholesterols (mg/dL)
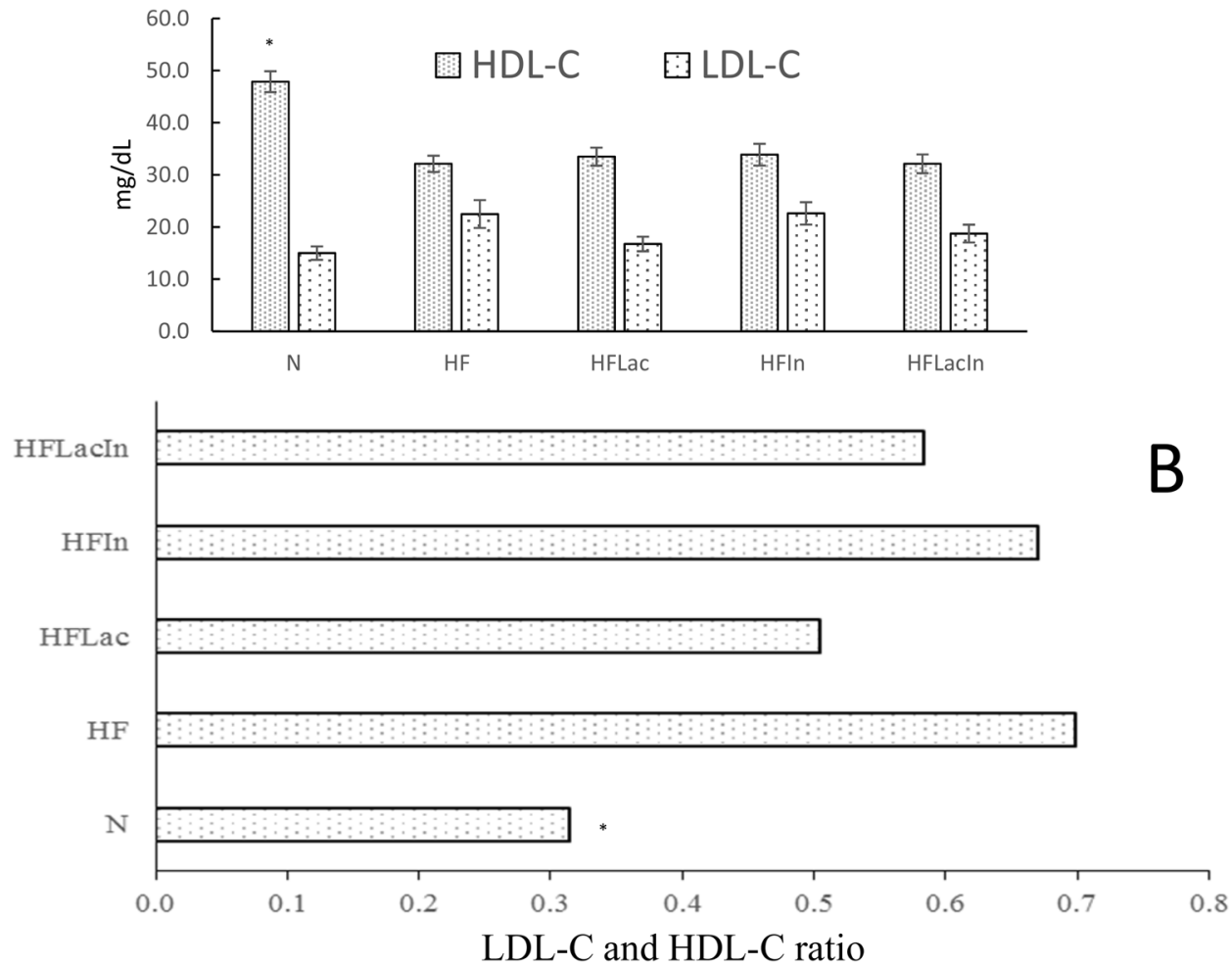

Figure 2. Serum HDL (mg/dL)- and LDL-cholesterol concentrations of the rats in each group ( $\mathrm{n}=13)$. (N) Rat fed a normal diet (HF) Rats fed with a high fat diet (HFLac, HFIn, HFLacIn) Rats fed with a high fat diet and $L$. acidophilus, inulin, and L. acidophilus + inulin, respectively. (A) Serum HDL-C and LDL-C concentrations and (B) the LDL-C/HDL-C ratio.

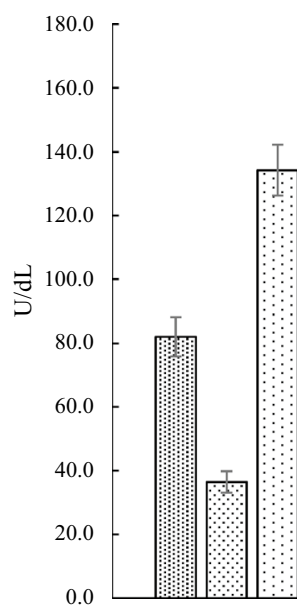

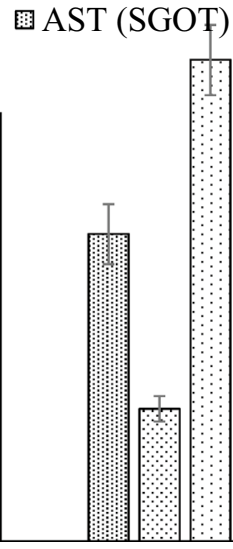

$\mathrm{HF}$

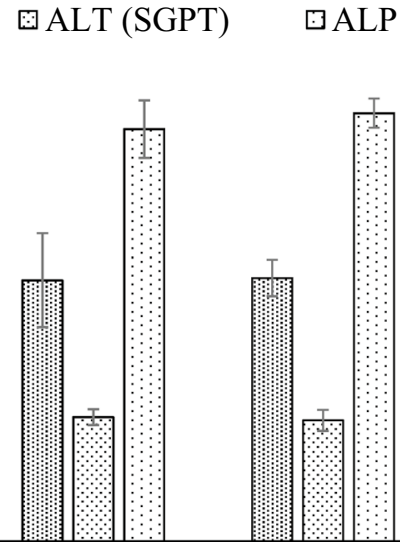

HFIn

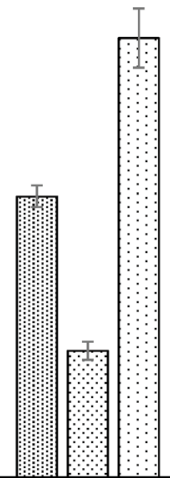

HFLacIn

Figure 3. Serum liver enzyme activities $(U / d L)$ of the rats in each group $(n=13)$. $(N)$ Rat fed a normal diet (HF) Rats fed with a high fat diet (HFLac, HFIn, HFLacIn) Rats fed with a high fat diet and L. acidophilus, inulin, and L. acidophilus + inulin, respectively. AST aspartate transaminase, ALT alanine transaminase, $A L P$ alkaline phosphatase. 


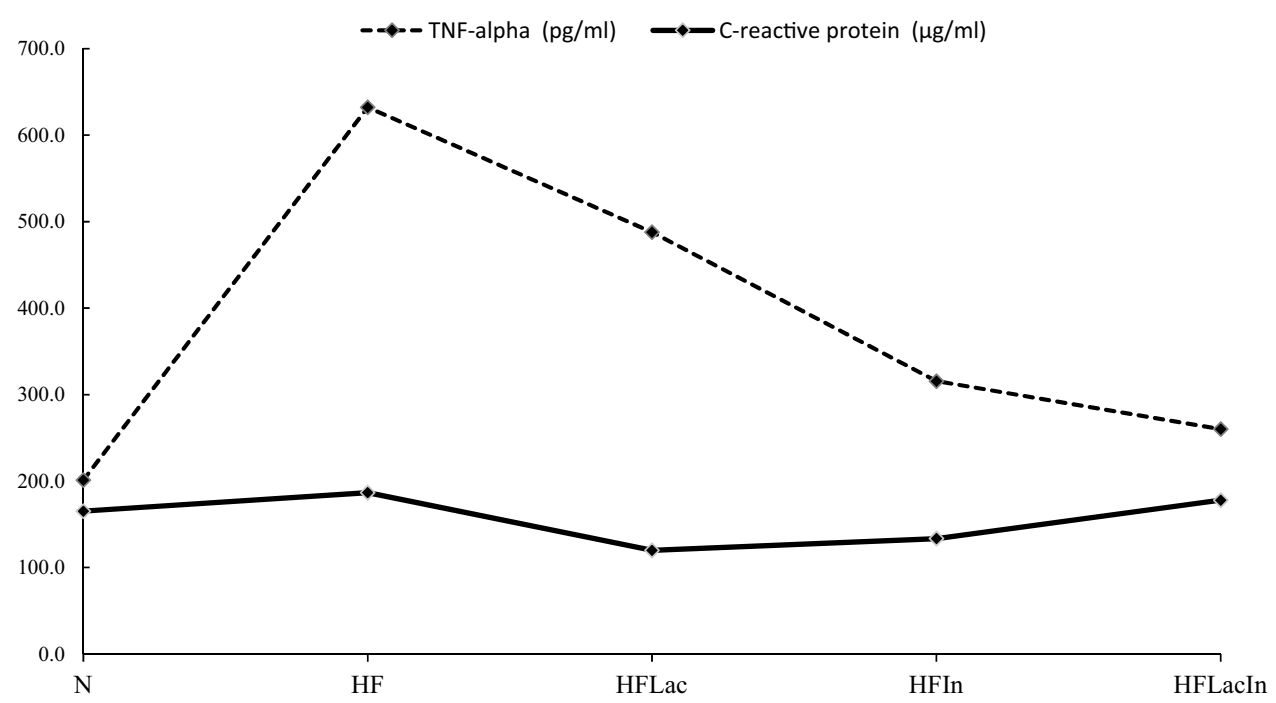

Figure 4. Serum concentrations of tumor necrosis factor-alpha (TNF-alpha; pg/ml) and C-reactive protein (CRP; $\mu \mathrm{g} / \mathrm{ml})$ of the rats in each group $(\mathrm{n}=13)$. (N) Rat fed a normal diet (HF) Rats fed with a high fat diet (HFLac, HFIn, HFLacIn). Rats fed with a high fat diet and L. acidophilus, inulin, and L. acidophilus + inulin, respectively.

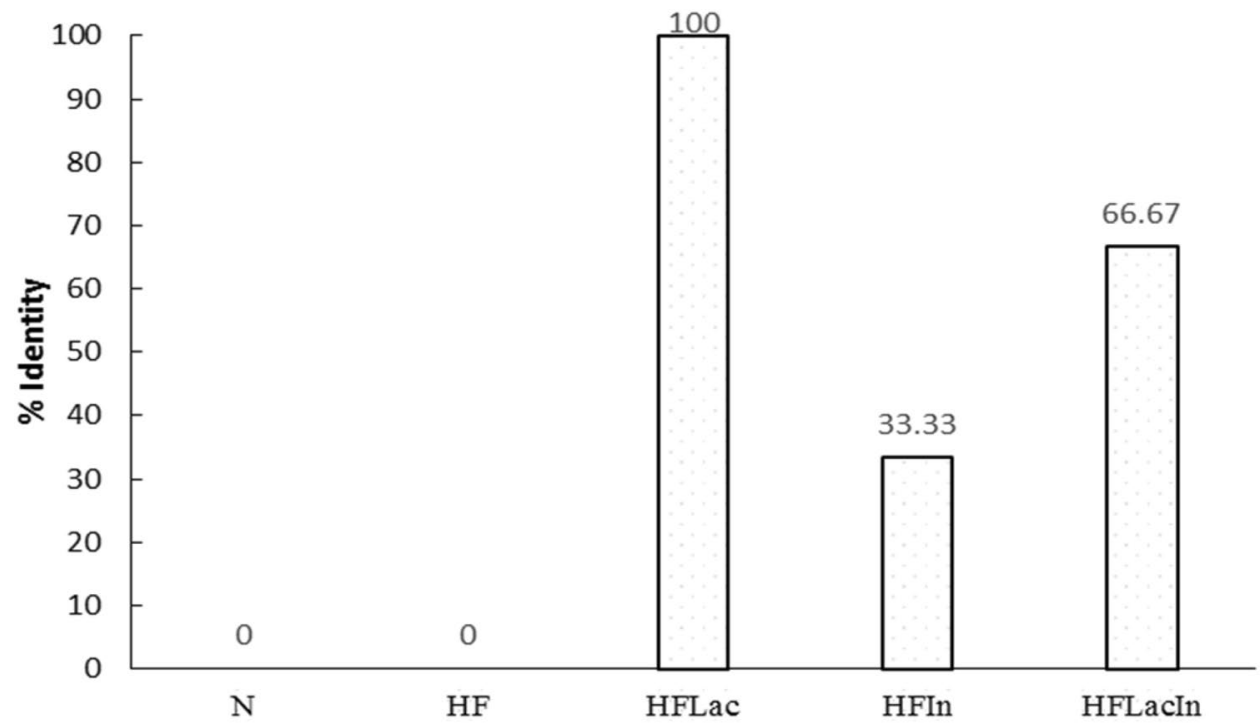

Figure 5. Presence of L. acidophilus in rat feces in each group $(\mathrm{n}=13)$. (N) Rat fed a normal diet (HF) Rats fed with a high fat diet (HFLac, HFIn, HFLacIn). Rats fed with a high fat diet and L. acidophilus, inulin, and $L$. acidophilus + inulin, respectively. \% identity values are shown.

in the HF group than in the N group (Fig. 4). However, the concentrations in the HFLac, HFIn, and HFLacIn groups were much lower than that in the HF group by $18.56-56.59 \%$. Rats administered the synbiotic combination had the lowest TNF- $\alpha$ concentration among the HF groups by $56.59 \%$. CRP is another serum marker of inflammation. The HF group exhibited a higher serum CRP concentration than the $\mathrm{N}$ group, and probiotic administration, but not prebiotic or synbiotic administration, reduced this by $35.71 \%$ when compared to HF group (Fig. 4).

Hepatocyte histopathology. Histological examination of the rat livers (Supplemental Fig. 1) revealed that the hepatocytes of the HF group contained considerable more and larger fat droplets than the $\mathrm{N}$ group. However, no significant histological differences were found among the four HF groups.

Lactobacillus identification in feces. We analyzed the feces of each rat group at the end of the study to identify the presence of $L$. acidophilus (Fig. 5). As expected, feces from the $\mathrm{N}$ and HF groups did not contain $L$. 


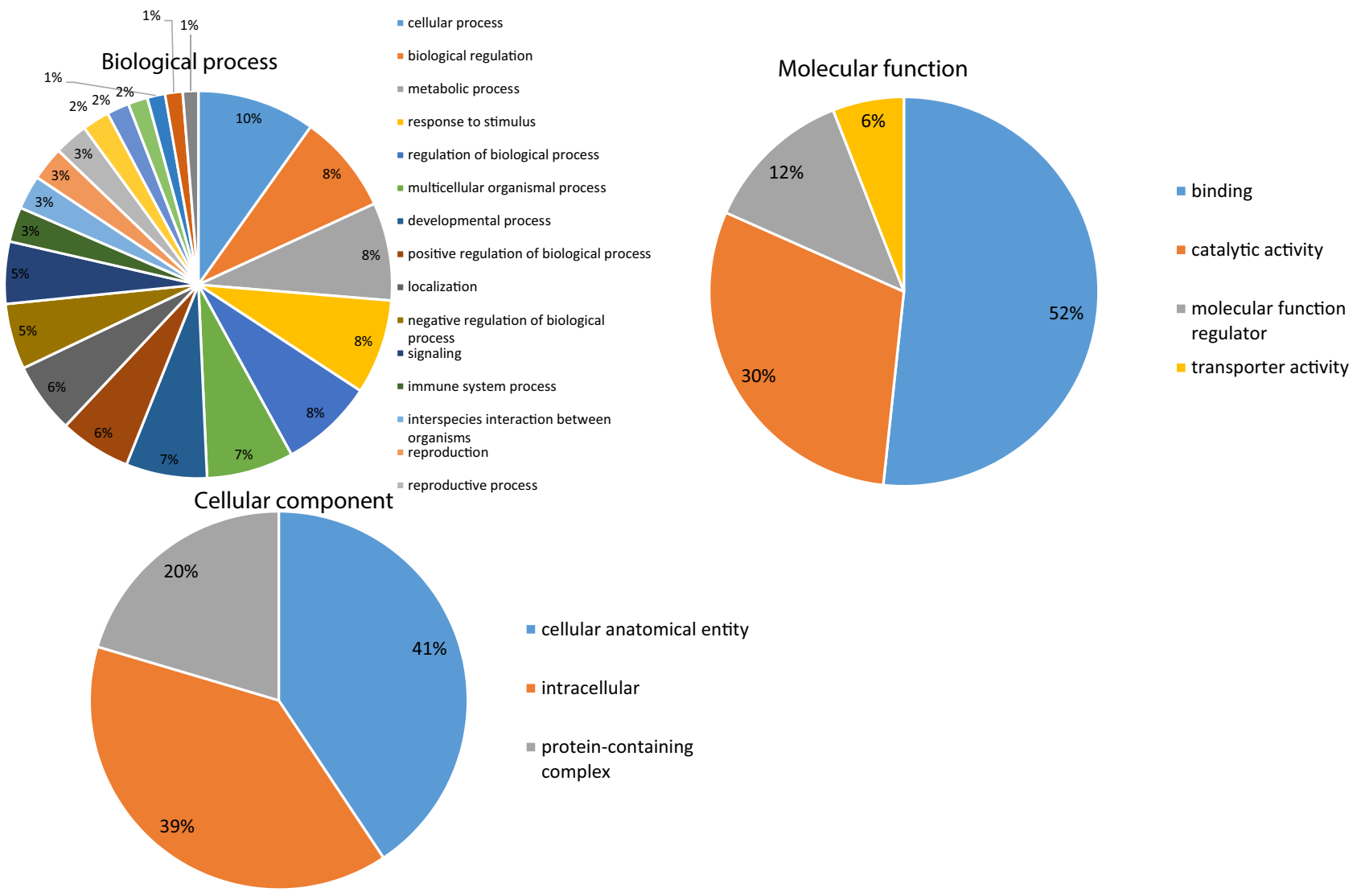

Figure 6. Gene ontology classification of hepatic proteins. The results of SDS-PAGE and MS analysis show the protein expression patterns of rats fed a high-fat diet (HF) and rats fed the high-fat diet and administered $L$. acidophilus (HFLac). $M$ molecular marker.

acidophilus. In contrast, the feces of every rat in the HFLac group contained L. acidophilus. Surprisingly, some of the fecal samples from rats of the HFLacIn group did not contain L. acidophilus. Furthermore, some of the fecal samples from the HFIn group contained L. acidophilus.

Hepatic proteome. The HFLac group was the group that showed the lowest LDL-C/HDL-C ratio and serum CRP concentration, and therefore we next separated the proteins in the livers of rats from the HF and HFLac groups by SDS-PAGE and stained the resulting gels using Coomassie blue (Supplemental Fig. 2). Fulllength gels are presented in Supplemental Fig. 3. Next, each lane was cut into small pieces, in-gel digestion was performed, and the peptide extracts were analyzed by MS. A total of 3,944 proteins were identified in the livers. The hepatic protein profiles of the HF and HFLac groups were compared, and 534 proteins were found to be upregulated and 308 downregulated in the HFLac group. GO classification of the differentially expressed proteins demonstrated that cellular process (10\%), binding (52\%), and cellular anatomical entity (41\%) were the major classes under the biological process, molecular function, and cellular component terms, respectively (Fig. 6). The top-20 most upregulated and downregulated proteins are presented in Tables 1 and 2, respectively.

Endoplasmin, 40S ribosomal protein S9, and alpha-1-inhibitor 3 were upregulated in the HFLac group, with fold differences of 5.75, 4.93, and 4.00, respectively. Additionally, ubiquitin, peroxiredoxin-5, and mitochondrial and dihydrofolate reductase were down-regulated in the HFLac group, with fold differences of 11.15, 5.92, and 4.88, respectively. The differentially expressed proteins were then subjected to pathway analysis using Blast2Go software: this showed that glutathione metabolism was the principal upregulated pathway in the HFLac group (Fig. 7). Specifically, glutathione S-transferase Mu 2 (GSTM2_RAT), glutathione S-transferase omega-1 (GSTO1_RAT), and phospholipid hydroperoxide glutathione peroxidase (GPX41_RAT) were upregulated in the HFLac group. In contrast, fatty acid elongation and glycerolipid metabolism were downregulated in the HFLac group (Fig. 8). In the fatty acid elongation pathway, sterol O-acyltransferase 2 (SOAT2_RAT), 3-hydroxyacylCoA dehydrogenase type-2 (HCD2_RAT), isovaleryl-CoA dehydrogenase (IVD_RAT), and short-chain specific acyl-CoA dehydrogenase (ACADS_RAT) were downregulated in the HFLac group. With respect to glycerolipid metabolism, lipoprotein lipase (LIPL_RAT) and pancreatic lipase-related protein 1 (LIPR1_RAT) were downregulated in the HFLac group (Fig. 8). Furthermore, the mitogen-activated protein kinase (MAPK) signaling pathway was downregulated in the HFLac group (Fig. 9). This finding is consistent with the much lower serum TNF- $\alpha$ concentration in the HFLac group than in the HF group. 


\begin{tabular}{|l|l|l|l|l|l|l|c|}
\hline Accession no & Protein & Fold difference & Score & M.W & pI & \% Cov & No. of peptides \\
\hline ENPL_RAT & Endoplasmin & 5.75 & 566 & 92,713 & 4.72 & 28.6 & 19 \\
\hline RS9_RAT & 40 S ribosomal protein S9 & 4.93 & 154 & 22,578 & 10.66 & 25.3 & 6 \\
\hline A1I3_RAT & Alpha-1-inhibitor 3 & 4.00 & 755 & 16,3670 & 5.7 & 24.2 & 31 \\
\hline APT_RAT & Adenine phosphoribosyltransferase & 3.59 & 73 & 19,533 & 6.17 & 38.3 & 5 \\
\hline HS90B_RAT & Heat-shock protein 90-beta & 3.50 & 420 & 83,229 & 4.97 & 24.7 & 16 \\
\hline COX2_RAT & Cytochrome c oxidase subunit 2 & 3.38 & 91 & 25,925 & 4.6 & 19.4 & 3 \\
\hline A1M_RAT & Alpha-1-macroglobulin & 3.00 & 177 & 16,7019 & 6.46 & 16.5 & 18 \\
\hline PHB_RAT & Prohibitin & 2.92 & 193 & 29,802 & 5.57 & 39 & 7 \\
\hline TERA_RAT & Transitional endoplasmic reticulum ATPase & 2.86 & 236 & 89,293 & 5.14 & 26.2 & 16 \\
\hline MUG1_RAT & Murinoglobulin-1 & 2.67 & 648 & 16,5221 & 5.68 & 21.3 & 28 \\
\hline HS90A_RAT & Heat-shock protein 90-alpha & 2.62 & 210 & 84,762 & 4.93 & 20.2 & 14 \\
\hline CO3_RAT & Complement C3 & 2.50 & 275 & 18,6342 & 6.12 & 18.9 & 23 \\
\hline ACTS_RAT & Actin, alpha skeletal muscle & 2.46 & 532 & 42,024 & 5.23 & 44.3 & 16 \\
\hline BUP1_RAT & Beta-ureidopropionase & 2.28 & 117 & 44,014 & 6.47 & 19.6 & 6 \\
\hline GSTM4_RAT & Glutathione S-transferase Yb-3 & 2.25 & 305 & 25,664 & 6.84 & 52.3 & 13 \\
\hline HMCS2_RAT & $\begin{array}{l}\text { Hydroxymethylglutaryl-CoA synthase, } \\
\text { mitochondrial }\end{array}$ & 2.21 & 851 & 56,876 & 8.86 & 46.3 & 22 \\
\hline UD16_RAT & UDP-glucuronosyltransferase 1-6 & 2.20 & 148 & 60,093 & 8.91 & 22.1 & 7 \\
\hline TPIS_RAT & Triosephosphate isomerase & 2.17 & 374 & 26,832 & 6.89 & 60.6 & 11 \\
\hline PGAM1_RAT & Phosphoglycerate mutase 1 & 2.00 & 121 & 28,814 & 6.67 & 37.4 & 7 \\
\hline AL1A7_RAT & Aldehyde dehydrogenase, cytosolic 1 & 2.00 & 115 & 54,525 & 7.1 & 23.4 & 10 \\
\hline
\end{tabular}

Table 1. Top-20 most upregulated hepatic proteins in the HFLac group vs. the HF group.

\begin{tabular}{|l|l|l|l|l|l|l|c|}
\hline Accession no & Protein & Fold difference & Score & M.W & pI & \% Cov & No. of peptides \\
\hline UBIQ_RAT & Ubiquitin & 11.15 & 216 & 8,560 & 6.56 & 78.9 & 7 \\
\hline PRDX5_RAT & Peroxiredoxin-5, mitochondrial & 5.92 & 436 & 22,165 & 8.94 & 75.6 & 13 \\
\hline DYR_RAT & Dihydrofolate reductase & 4.88 & 136 & 21,624 & 6.77 & 28.3 & 5 \\
\hline RS3A_RAT & 40 ribosomal protein S3a & 4.73 & 210 & 29,926 & 9.75 & 44.3 & 12 \\
\hline PHS_RAT & Pterin-4-alpha-carbinolamine dehydratase & 3.90 & 150 & 11,992 & 6.28 & 45.2 & 4 \\
\hline RL23A_RAT & 60S ribosomal protein L23a & 3.63 & 192 & 17,684 & 10.44 & 25.6 & 5 \\
\hline RL17_RAT & 60S ribosomal protein L17 & 3.44 & 196 & 21,383 & 10.2 & 29.9 & 5 \\
\hline EST5_RAT & Liver carboxylesterase B-1 & 3.40 & 121 & 62,455 & 6.25 & 25.3 & 10 \\
\hline EST4_RAT & Liver carboxylesterase 4 & 3.40 & 102 & 62,269 & 6.29 & 17.5 & 6 \\
\hline PDIA3_RAT & Protein disulfide-isomerase A3 & 3.33 & 631 & 56,588 & 5.88 & 39.2 & 17 \\
\hline CH10_RAT & 10 kDa heat-shock protein, mitochondrial & 3.28 & 380 & 10,895 & 8.89 & 84.3 & 11 \\
\hline ECHA_RAT & $\begin{array}{l}\text { Trifunctional enzyme subunit alpha, mito- } \\
\text { chondrial }\end{array}$ & 3.00 & 216 & 82,613 & 9.16 & 24.6 & 16 \\
\hline ARG11_RAT & Arginase-1 & 2.85 & 202 & 34,951 & 6.76 & 39.9 & 9 \\
\hline RS18_RAT & 40 ribosomal protein S18 & 2.67 & 267 & 17,708 & 10.99 & 40.1 & 8 \\
\hline RS10_RAT & 40 Sibosomal protein S10 & 2.38 & 239 & 18,904 & 10.15 & 33.3 & 6 \\
\hline RLA1_RAT & 60S acidic ribosomal protein P1 & 2.30 & 63 & 11,491 & 4.28 & 28.9 & 2 \\
\hline COF1_RAT & Cofilin-1 & 2.22 & 107 & 18,521 & 8.22 & 41 & 5 \\
\hline MDHC_RAT & Malate dehydrogenase, cytoplasmic & 2.21 & 314 & 36,460 & 6.16 & 33.5 & 11 \\
\hline ATPD_RAT & ATP synthase subunit delta, mitochondrial & 2.21 & 111 & 17,584 & 5.16 & 18.5 & 3 \\
\hline CDC42_RAT & Cell division control protein 42 homolog & 2.13 & 63 & 21,245 & 6.15 & 15.7 & 2 \\
\hline
\end{tabular}

Table 2. Top-20 most downregulated hepatic proteins in the HFLac group vs. the HF group.

\section{Discussion}

The feeding of a high-fat diet for 4 weeks increased the serum LDL-cholesterol concentration, reduced that of HDL-cholesterol, and abnormal hepatic fat droplets accumulation in the rats, which is consistent with dyslipidemia and fatty liver. Dyslipidemia is a well-known major risk factor for cardiovascular disease, which is a leading cause of morbidity and mortality worldwide ${ }^{2}$. Dietary supplementation with probiotics, prebiotics, or synbiotics has been suggested to improve cholesterol metabolism, but inconsistent findings have been made in both animal and human studies ${ }^{17}$. Therefore, in the present study, we assessed the effects of oral supplementation with an $L$. 


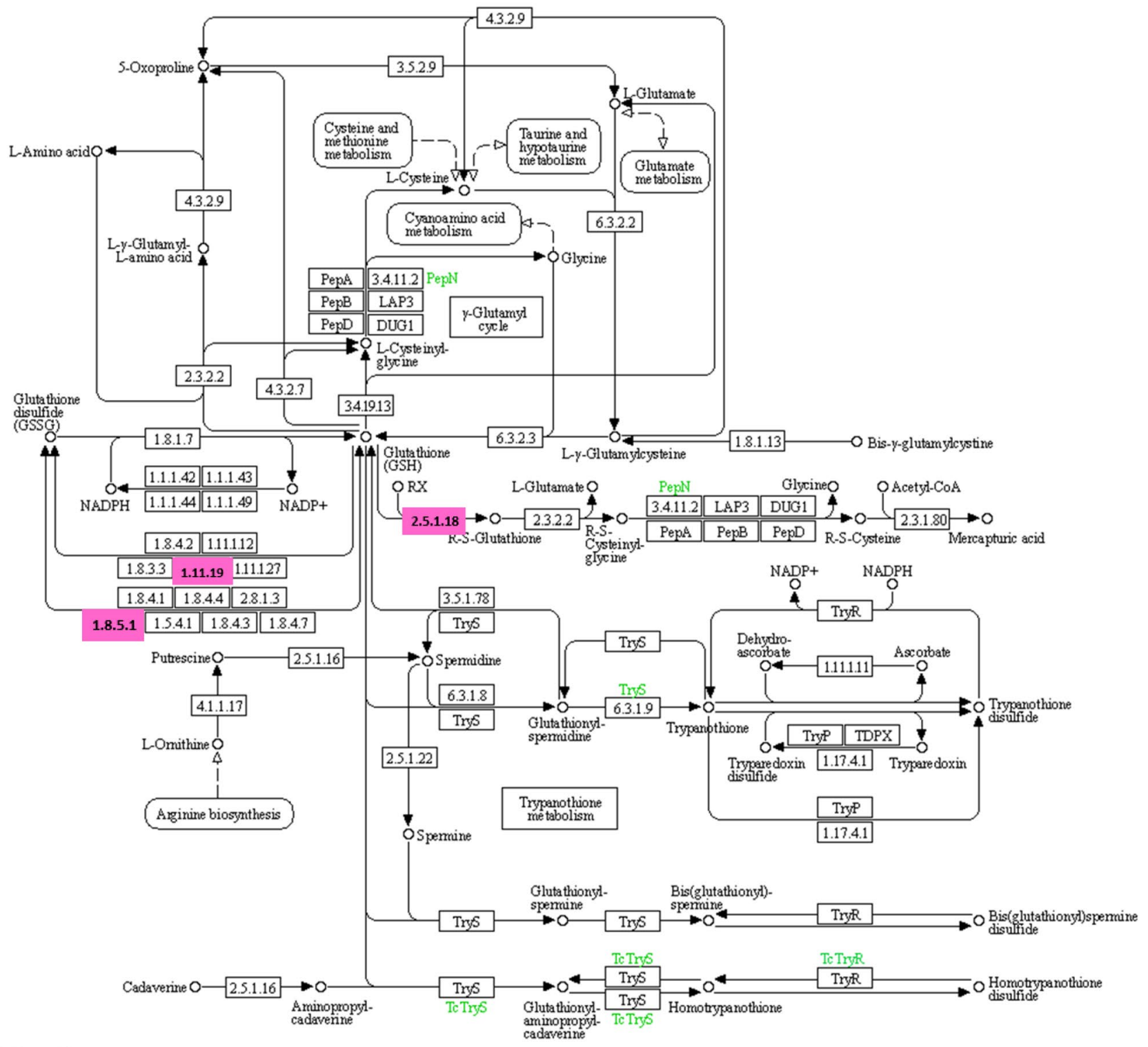

00480411420.

Figure 7. Glutathione metabolism. Pink boxes: upregulated proteins in the HFLac group.

acidophilus probiotic, inulin, as a prebiotic, and the combination of L. acidophilus and inulin, as a synbiotic, in rats fed a high-fat diet.

The results demonstrate that these supplements reduce serum LDL-cholesterol in rats, and in particular, rats that were administered Lactobacillus (HFLac) had a superior LDL/HDL ratio than rats administered the other supplements. These findings were consistent with those of a previous study ${ }^{18}$, which showed that mice fed a high-fat diet and administered L. acidophilus $\left(4 \times 10^{7} \mathrm{cfu} / \mathrm{mL}\right)$ for 10 weeks had significantly lower TC and LDL-cholesterol concentrations. Furthermore, the present proteomic study demonstrated that microsomal fatty acid elongation and glycerolipid metabolism are downregulated by L. acidophilus intake. Microsomal fatty acid elongation is considered to be the predominant pathway for the elongation of fatty acid with $\geq 12$ carbon atoms ${ }^{19}$. This pathway uses fatty acids derived from the diet, and its inhibition is associated with lower plasma lipid concentrations ${ }^{20}$. Therefore, the downregulation of fatty acid elongation in the HFLac group may contribute to the low circulating LDL-cholesterol concentration. In the glycerolipid metabolism category, lipase expression was downregulated in the HFLac group. Lipases are a subclass of the esterases that play important roles in the digestion, transport, and processing of dietary lipids ${ }^{21}$; therefore, inhibition of lipase activity could reduce the gastrointestinal absorption of fats, leading to their loss in the feces, rather than their full absorption ${ }^{22}$, and also explain the low serum LDL-cholesterol concentration. Even though our proteomic analysis changes are pointing 


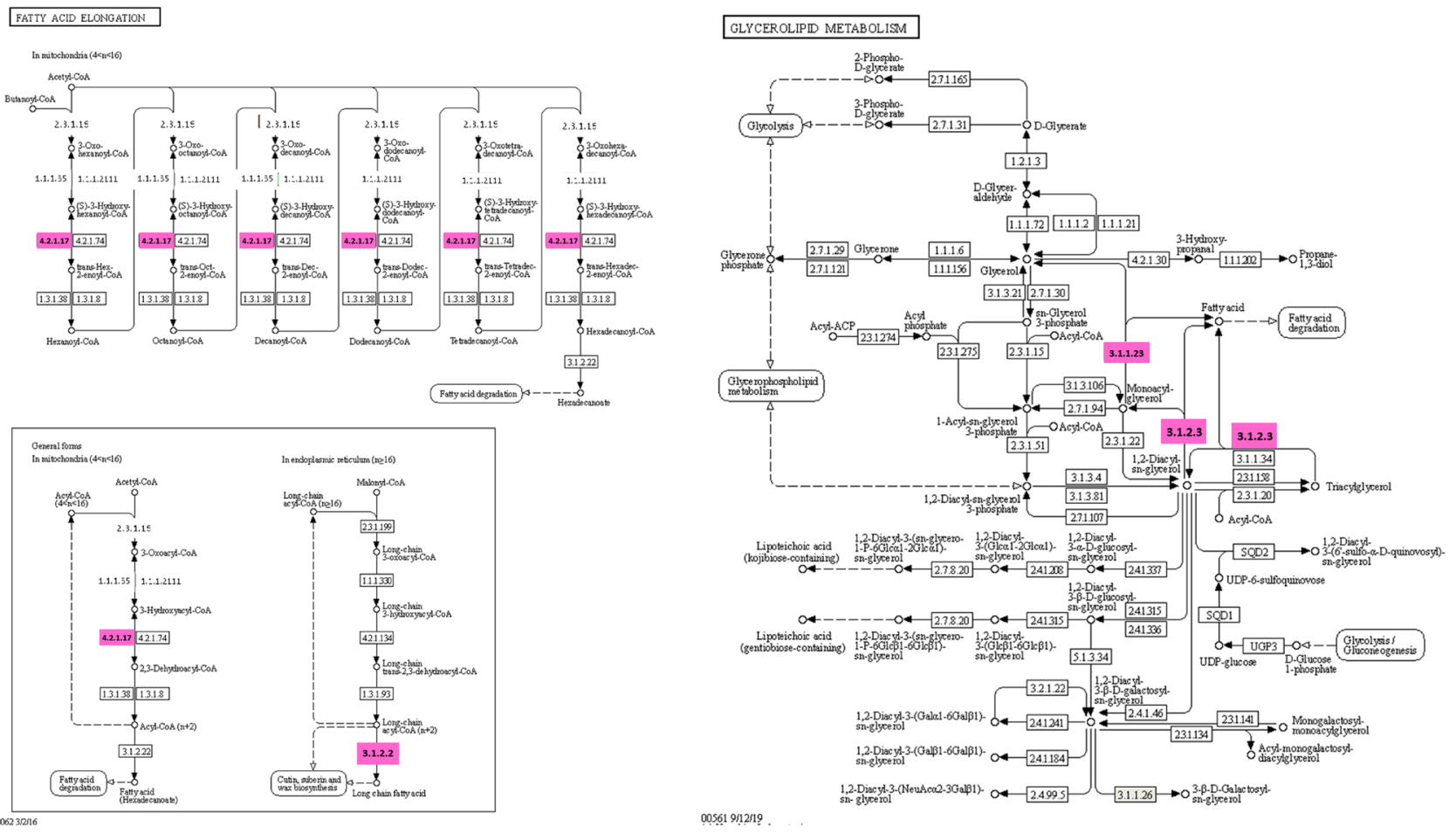

Figure 8. Fatty acid elongation and glycerolipid metabolism. Pink boxes: downregulated proteins in the HFLac group.

in the same direction of our serum lipid profile and inflammation, it was our limitation that proteomics results should confirmation with molecular methods like western blotting.

Inulin is a form of soluble fiber that not digested in the human small intestine, but can be broken down by intestinal bacteria, which generates products that are beneficial for health ${ }^{23}$. The addition of inulin to the diet at a low concentration $(0.04 \%)$ has been shown to considerably improve the growth of L. acidophilus ${ }^{24}$; therefore, we also compared the effects of Lactobacillus administration alone with that of this prebiotic and a combination of the two (a synbiotic) in the present study. Inulin consumption reduces circulating TC and LDL-cholesterol concentration $s^{25}$ by increasing the deconjugation of bile acids by gut bacteria ${ }^{26}$. This may have been one of the mechanisms whereby inulin reduced LDL-cholesterol in the present study.

Effects of Lactobacillus probiotics on immune regulation have been previously described. Certain probiotic strains have positive effects on immunity because they stimulate host immune responses ${ }^{27}$. TNF- $\alpha$ and CRP regulate the immune system and protect the body from microbiological invasion by activating the inflammatory response. Increases in the concentration of either TNF- $\alpha$ or CRP have also been reported alongside dyslipidemia and obesity ${ }^{28}$. In the present study, we found that the TNF- $a$ concentrations in the HFLac, HFIn, and HFLacIn groups were less than that in the HF group. Furthermore, the CRP concentration was lower in the HFLac group than in the HF group. Therefore, the effects of L. acidophilus on immunity may be mediated at least in part through reductions in the concentrations of TNF- $\alpha$ and CRP.

L. acidophilus has also been shown to increase the levels of cluster of differentiation (CD) $4+, \mathrm{CD} 4+/ \mathrm{CD} 8+$, and immunoglobulin A, while significantly reducing those of interleukin (IL)- 6 and TNF- $\alpha$ in vitro and in vivo ${ }^{27}$. The present proteomics study showed that glutathione metabolism was an upregulated pathway in the HFLac group. Glutathione (GSH) plays important roles in the defense against oxidative stress. The cysteine residues of GSH are readily oxidized to form glutathione disulfide (GSSG) by free radicals and reactive oxygen/nitrogen species $^{29}$. We also found the relationship of oxidation reduction and cellular biosynthetic process (Supplemental Fig. 4.). Therefore, the consumption of L. acidophilus may reduce oxidative stress in rats through the glutathione pathway. A reduction in oxidative stress would reduce inflammation and the secretion of the inflammatory cytokine, TNF- $\alpha$, and indeed the circulating TNF- $\alpha$ concentrations in the HFLac, HFIn, and HFLacIn groups were much lower than those in HF rats.

Proteins of the MAPK signaling pathway were also found to be differentially expressed in the proteomic analysis. This pathway can be activated by TNF- $\alpha$, and the lower serum TNF- $\alpha$ concentration of the HFLac group occurred alongside the downregulation of 12 proteins in the MAPK signaling pathway. The downregulation of this pathway may be associated with lower inflammation in tissues.

Some previous studies have also shown positive effects of inulin on the immune system; for example, it increases natural killer (NK) cell activity both in mice and healthy elderly people ${ }^{30,31}$. Additionally, another study showed that oxidative stress was ameliorated by inulin supplementation in a rat model of type 2 diabetes mellitus $^{32}$. However, inulin showed little capacity to suppress IL- 6 and TNF- $\alpha$ secretion in lipopolysaccharidetreated murine macrophages ${ }^{33}$. To our knowledge, a clear mechanism for the effects of inulin on the immune 


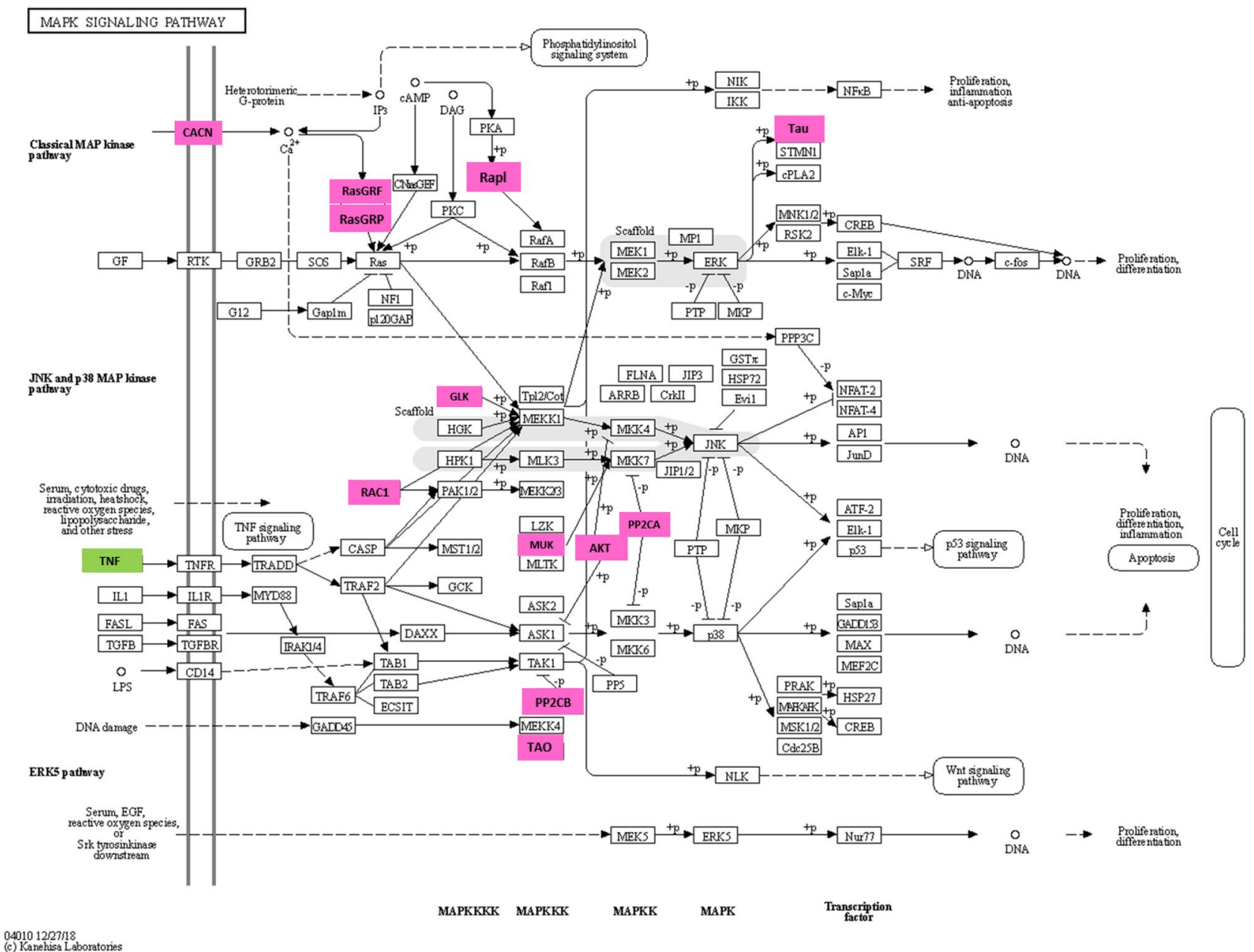

Figure 9. MAPK signaling pathway. Green box: tumor necrosis factor-alpha. Pink boxes: downregulated proteins in the HFLac group.

system has yet to be identified, but it might involve reductions in inflammatory mediators. In the present study, the HFLacIn group did not show superior effects to those of probiotic (HFLac group) or prebiotic (HFIn group) alone. This might be because of the administration of an inappropriate ratio of inulin and L. acidophilus in the HFLacIn group; this is because it has been suggested that synbiotics should induce a synergistic effect of their pro- and prebiotic components. Prebiotics should not only stimulate the growth of probiotic microbes, but also their survival ${ }^{34}$.

The presence of $L$. acidophilus was confirmed by RT-PCR analysis of the rat feces in the present study. The World Health Organization defines probiotics as living microorganisms that when administered in adequate amounts may provide health benefits for the host ${ }^{7}$. In the present study, we found that the feces of the $\mathrm{N}$ and HF groups did not contain L. acidophilus, as expected. One hundred percent of the feces of the HFLac group contained L. acidophilus, whereas only $66.7 \%$ of HFLacIn group did so, which might be the result of an inappropriate ratio of $L$. acidophilus and inulin administration. Furthermore, surprisingly, feces from some of the rats in the HFIn group contained L. acidophilus, which is consistent with the previous discovery of L. acidophilus in normal rat feces ${ }^{35}$, and suggests that the inulin was beneficial for the survival of L. acidophilus in the rat gastrointestinal system.

In conclusion, the probiotic, prebiotic, and synbiotic used in the present study may represent natural means of maintaining hypocholesterolemia. Our findings suggest that the mechanisms of the beneficial effects of $L$. acidophilus in rats involve reductions in lipid elongation and glycerolipid metabolism, activation of antioxidant mechanisms, and less inflammation because of downregulation of the a mitogen-activated protein kinase (MAPK) signaling pathway. A fascinating future research should be investigated more detail dose-dependent manner.

\section{Methods}

Preparation of probiotics and diets. L. acidophilus was obtained in freeze-dried form (Natural Organic laboratories, Inc.,: Amityville, NY, USA) at a concentration of $4 \times 10^{7}$ colony-forming units per gram (CFU/g) and stored in opaque packaging at $-20^{\circ} \mathrm{C}$. Inulin was obtained from COSUCRA: Warcoing Belgium. The rat diets were prepared according to the method of Udomkasemseb and colleagues ${ }^{6}$. The proportions of casein, corn 
starch, DL-methionine, mineral and vitamin mixtures, choline bitartrate, and corn oil were the same in each diet, and those of sucrose, cellulose, lard, and inulin were varied. The sucrose and lard contents of the high-fat diets were $33 \%$ and $17 \%$, respectively. Inulin was included in the high-fat diet by replacing cellulose, to $1 \%$ of the total. The energy content of the normal diet was $1610 \mathrm{~kJ} / \mathrm{g}$, comprising carbohydrate $(\mathrm{C}) 67.53 \%$, protein $(\mathrm{P})$ $20.78 \%$, and fat (F) $11.69 \%$ per unit mass. The energy content of the high-fat diets was $1966 \mathrm{~kJ} / \mathrm{g}$, comprising C $40.85 \%$, P $17.02 \%$, and F $42.14 \%$. Fresh diet was provided for the rats at 10:00 every day. Probiotic was orally administered with $1.5 \mathrm{ml}$ of $L$. acidophilus to the appropriate groups by reconstituted $1 \mathrm{~g}$ of probiotics in $1.5 \mathrm{ml}$ of $0.9 \%$ sodium chloride solution $\left(4 \times 10^{7} \mathrm{cfu} / \mathrm{ml}\right)$, and the other groups were administered $1.5 \mathrm{ml}$ of sodium chloride solution per rat.

Animals and experimental design. The animal study protocol was approved by the Faculty of Tropical Medicine Animal Care and Use Committee, Mahidol University with the approval number FTM-ACUC 023/2561 and in compliance with the ARRIVE guidelines. All methods were carried out in accordance with relevant guidelines and regulations. The sample size was calculated using the formula of $\mathrm{n}=\left[\left(Z_{1-\alpha / 2}+Z_{1-\beta}\right)^{2}\left(\sigma_{1}{ }^{2}+\right.\right.$ $\left.\left(\left[\sigma_{2}^{2} / \mathrm{r}\right]\right)\right] / \delta^{2} \mathrm{n}=$ sample size, $\sigma=$ standard deviation, $\mathrm{r}=\mathrm{n}_{2} / \mathrm{n}_{1}, \delta=$ difference between two group of means, power $(1-\beta)=0.9$ and type I error $(\alpha)=0.01$. Ninety-one 5 -week-old male Sprague-Dawley rats, weighing approximately 100-120 g, were obtained from Nomura Siam International (Bangkok, Thailand). Health certificate of rats was issued by M Clea Bioresources Co., Ltd (Bangkok, Thailand). The rats were acclimated to their new surroundings for 7 days with 2 or 3 rats housed per cage, during which period standard rat chow and water were available ad libitum. After that, the rats were simple randomly allocated by chance to five groups $(\mathrm{n}=13$ each): the rats fed with a normal diet $(\mathrm{N})$, rats fed with a high fat diet (HF), rats fed with a high fat diet and inulin (HFIn), rats fed with a high fat diet and L. acidophilus (HFLac) and rats fed with a high fat diet, L. acidophilus and inulin (HFLacIn), which were fed ad libitum for 30 days. The groups being compared, are including $\mathrm{N}$ and $\mathrm{HF}$ groups. Inclusion and exclusion criteria were not set during the experiment. To minimize potential confounders between the groups, the cage of each treatments were separately located in sequence. The food consumption and body mass of the rats was measured weekly. Feces were collected on day 30 for Lactobacillus identification. At the end of the experimental period, the rats were fasted overnight for around 12-16 h and were then euthanized using carbon dioxide, according to the procedure of the Laboratory Animal Science Unit of the Faculty of Tropical Medicine. Blood was collected subsequently from the vena cava for biochemical analysis and the measurement of inflammatory marker concentrations. Livers were collected, weighed, and prepared for histological and proteomic analyses.

Biochemical analyses and measurement of inflammatory marker concentrations. Three milliliters of whole blood were collected from each rat into potassium-EDTA tubes for immediate measurement of the white blood cell count using automated Cobas 6800/8800 systems (Roche Group, Switzerland). Additional blood was collected into plain tubes and centrifuged at $3000 \times g$ and $5{ }^{\circ} \mathrm{C}$ for $15 \mathrm{~min}$. The obtained serum was stored at $-80^{\circ} \mathrm{C}$ until use. One milliliter of serum per rat was used for lipid profiling (TC, TG, LDL-cholesterol, and HDL-C), the assessment of renal function (blood urea nitrogen (BUN) and creatinine concentrations), and liver enzyme activity measurement (alanine aminotransaminase (ALT), aspartate transaminase (AST), and alkaline phosphatase (ASP)) using the Cobas 6800/8800 systems. Two hundred microliters of serum were used to measure the concentrations of tumor necrosis factor-alpha (TNF- $\alpha$ ) (Abcam: Cambridge, UK) and c-reactive protein (CRP) (Sigma-Aldrich, Inc.: St. Louis, MO, USA) using rat elisas, according to the manufacturers' instructions.

Histological examination. The left lateral lobes of the rat livers were sectioned transversely and fixed in $10 \%$ buffered formalin for $48 \mathrm{~h}$. The livers were then embedded in paraffin, sectioned at $5.0 \mu \mathrm{m}$ thickness, and stained with hematoxylin and eosin, prior to examination under a light microscope (Zeiss Imager.M2, Oberkochen, Germany).

Proteomic analysis. Rat liver samples from the same group were pooled, frozen in liquid nitrogen, and ground using a mortar. Lysis buffer containing 1\% SDS (Merck, Germany), 1\% triton X-100 (Merck) and 1\% nacl (Merck) was then added and lysates were prepared using an ultrasonicator (Sonics \& Materials, USA). The lysates were then centrifuged at $12,000 \times \mathrm{g}$ for $5 \mathrm{~min}$ at $4{ }^{\circ} \mathrm{C}$, the supernatants were collected, and their protein concentrations were measured using a Quick Start Bradford Protein Assay (Bio-Rad, USA). Lysate aliquots containing $30 \mu \mathrm{g}$ of protein were separated by $12 \%$ SDS-PAGE (Bio-Rad) and the gels generated were stained using Coomassie G-250 solution (Bio-Rad). Each lane was then cut into 13 pieces and the proteins within subjected to tryptic in-gel digestion ${ }^{16}$. The trypsin-digested fractions were separated using an Ultimate 3000 Nano-LC system (Dionex; Surrey, UK) at a flow rate of $300 \mathrm{nl} / \mathrm{min}$. Mobile phase A was $2 \%(\mathrm{v} / \mathrm{v})$ acetonitrile and $0.1 \%(\mathrm{v} / \mathrm{v})$ formic acid in HPLC-grade water and mobile phase B was $0.1 \%(\mathrm{v} / \mathrm{v})$ formic acid in HPLC-grade acetonitrile. The peptides were then analyzed using a micrOTOF QII mass spectrometer (MS) (Bruker; Bremen, Germany) and Hystar software. The MS and MS/MS spectra were acquired in $m / z$ ranges of 400-2000 and 50-1500, respectively.

Proteomic data analysis. The raw MS data were converted into a Mascot generic file (.mgf) using data analysis software (Mascot v.2.3.0; Matrix Science, London, UK), and this was used for protein identification using the Swissprot database. The parameters were set to Mus musculus (taxonomy), trypsin (enzyme), one (missed cleavage site), 1.2 Da (mass tolerance for the precursor), and Swissport 0.6 Da (mass tolerance for fragment ions). The oxidation of methionine and carbamidomethylation of cysteine residues were allowed as 
modifications. Only proteins with $95 \%$ confidence were reported. The exponentially modified protein abundance index (empai) was used for protein semi-quantification. Protein profiles were classified according to gene ontology (GO) using Blast2Go software and pathway analysis was performed using the Kyoto Encyclopedia of Genes and Genomes (KEGG) database ${ }^{36,37}$.

Lactobacillus identification. Fresh fecal samples from three rats per group were collected and stored at $-80^{\circ} \mathrm{C}$ until analyzed. DNA was extracted using a qiaamp DNA stool mini kit (Qiagen, USA) from $100 \mathrm{mg}$ feces and its purity was assessed using a nanodrop 2000 spectrophotometer (Thermo Fisher Scientific, USA). DNA from L. acidophilus was identified by real-time PCR amplification using itaq Universal SYBR Green Supermix (Bio-Rad). The reaction mixtures comprised $12 \mu \mathrm{l}$ of SYBR Green Supermix, $2 \mu \mathrm{l}$ each of the forward and reverse primer $(10 \mu \mathrm{m})$, and $6.0 \mu \mathrm{l}$ nuclease-free water. Two microliters of DNA samples were then added to each reaction mixture and amplified by thermal cycler (C1000 Touch, Applied Biosystems, USA) using an initial activation step $\left(95^{\circ} \mathrm{C}\right.$ for $5 \mathrm{~min}$ ), followed by 40 cycles of denaturation $\left(95^{\circ} \mathrm{C}\right.$ for $15 \mathrm{~s}$ ), annealing $\left(57^{\circ} \mathrm{C}\right.$ for $15 \mathrm{~s}$ ), and extension $\left(60^{\circ} \mathrm{C}\right.$ for $\left.15 \mathrm{~s}\right)$; and a final step of $65^{\circ} \mathrm{C}$ for $5 \mathrm{~s}$ and $95^{\circ} \mathrm{C}$ for $5 \mathrm{~s}$. The forward and reverse primer sequences $\left(5^{\prime}-3^{\prime}\right)$ for $L$. Acidophilus detection were TGCAAAGTGGTAGCGTAAGC and CCTTTCCCT CACGGTACTG, respectively.

Statistical analysis. Body mass, biochemical parameters, inflammatory marker concentrations, and fecal Lactobacillus data are presented as means \pm standard deviations (SD) after assessing for normality with Shapiro-Wilk test. Data were analyzed using one-way analysis of variance (ANOVA) and differences between groups were further analyzed using Duncan's multiple range tests. Statistical significance was accepted when $P<0.05$. Statistical analysis was performed using SPSS Statistics software Version 18 (IBM Inc., Armonk, NY, USA).

\section{Data availability}

The data and materials supporting this article are available in faculty of tropical medicine, Mahidol University, Thailand.

Received: 22 November 2020; Accepted: 2 March 2021

Published online: 17 March 2021

\section{References}

1. World Health Organization. Obesity and Overweight. Preprint https://www.who.int/news-room/fact-sheets/detail/obesity-andoverweight (2020).

2. Feingold, K.R. \& Grunfeld, C. Obesity and dyslipidemia. in Endotext (ed. Feingold, K.R. et al.) (South Dartmouth, 2020).

3. Lira, F. S., Rosa-Neto, J. C., Antunes, B. M. \& Fernandes, R. A. The relationship between inflammation, dyslipidemia and physical exercise: From the epidemiological to molecular approach. Curr. Diabetes Rev. 10, 391-396 (2014).

4. Stelmach-Mardas, M. et al. Link between food energy density and body weight changes in obese adults. Nutrients. 8, 229 (2016).

5. Ngamlerst, C. et al. The potential of antioxidant-rich Maoberry (Antidesma bunius) extract on fat metabolism in liver tissues of rats fed a high-fat diet. BMC Complement. Altern. Med. 19, 294 (2019).

6. Udomkasemsab, A. \& Prangthip, P. High fat diet for induced dyslipidemia and cardiac pathological alterations in Wistar rats compared to Sprague Dawley rats. Clin. Invest. Arterioscler. 31, 56-62 (2019).

7. Food and Agriculture Organization of the United \& World Health Organization. Probiotics in food: Health and nutritional properties and guidelines for evaluation. in Report of a Joint FAO/WHO Expert Consultation on Evaluation of Health and Nutritional Properties of Probiotics in Food including Powder Milk with Live Lactic Acid Bacteria. Preprint http://www.fao.org/3/a-a0512e.pdf (2002).

8. Matsumoto, M., Kurihara, S., Kibe, R., Ashida, H. \& Benno, Y. Longevity in mice is promoted by probiotic-induced suppression of colonic senescence dependent on upregulation of gut bacterial polyamine production. PLoS ONE 6, e23652 (2011).

9. De Rodas, B. Z., Gilliland, S. E. \& Maxwell, C. V. Hypocholesterolemic action of Lactobacillus acidophilus ATCC 43121 and calcium in swine with hypercholesterolemia induced by diet. J. Dairy Sci. 79, 2121-2128 (1996).

10. Maxwell, C., Nelson, C. R. \& Gilliland, S. E. Assimilation of cholesterol by Lactobacillus acidophilus. Appl. Environ. Microbiol. 49 (2), 377-381 (1985).

11. de Sales Guilarducci, J., Marcelino, B.A.R., Konig, I.F.M., Orlando, T.M., Varaschin, M.S. \& Pereira, L.J. Therapeutic effects of different doses of prebiotic (isolated from Saccharomyces cerevisiae) in comparison to n-3 supplement on glycemic control, lipid profiles and immunological response in diabetic rats. Diabetol. Metab. Syndr.12, 69 (2020).

12. U.S. Department of Health and Human Services Food and Drug Administration Center for Food Safety and Applied Nutrition. The Declaration of Certain Isolated or Synthetic Non-Digestible Carbohydrates as Dietary Fiber on Nutrition and Supplement Facts Labels: Guidance for Industry. 2018. Preprint https://www.fda.gov/media/113663/download (2018). Accessed 20 Aug 2020.

13. Zhang, X., Hu, P., Zhang, X. \& Li, X. Chemical structure elucidation of an inulin-type fructan isolated from Lobelia chinensis lour with anti-obesity activity on diet-induced mice. Carbohydr Polym. 240, 116357 (2020).

14. Swanson, K. S. et al. The International Scientific Association for Probiotics and Prebiotics (ISAPP) consensus statement on the definition and scope of synbiotics. Nat. Rev. Gastroenterol. Hepatol. 17, 687-701 (2020).

15. Tang, T. et al. A synbiotic consisting of Lactobacillus plantarum S58 and hull-less barley $\beta$-glucan ameliorates lipid accumulation in mice fed with a high-fat diet by activating AMPK signaling and modulating the gut microbiota. Carbohydr. Polym. 243, 116398 (2020).

16. Reamtong, O. et al. Proteomic analysis of adult Schistosoma mekongi somatic and excretory-secretory proteins. Acta. Trop. 202, 105247 (2020).

17. Pandey, K. R., Naik, S. R. \& Vakil, B. V. Probiotics, prebiotics and synbiotics-A review. J. Food Sci. Technol. 52, 7577-7587 (2015).

18. Song, M. et al. Effect of Lactobacillus acidophilus NS1 on plasma cholesterol levels in diet-induced obese mice. J. Dairy Sci. 98, 1492-1501 (2015).

19. Cinti, D. L., Cook, L., Nagi, M. N. \& Suneja, S. K. The fatty acid chain elongation system of mammalian endoplasmic reticulum. Prog. Lipid. Res. 31, 1-51 (1992).

20. Hussain, M. M. \& Bakillah, A. New approaches to target microsomal triglyceride transfer protein. Curr. Opin. Lipidol. 19, 572-578 (2008).

21. Svendsen, A. Lipase protein engineering. Biochim. Biophys. Acta. 1543, 223-228 (2000). 
22. Aronne, L. J. Treating obesity: A new target for prevention of coronary heart disease. Prog. Cardiovasc. Nurs. 16, 98-106 (2001). 23. Shoaib, M. et al. Inulin: Properties, health benefits and food applications. Carbohydr. Polym. 20, 444-454 (2016).

24. Oliveira, R. P., Patrizia, O., Oliverira, M. N. \& Converti, A. Effect of inulin as prebiotic and synbiotic interactions between probiotics to improve fermented milk firmness. J. Food Eng. 107, 36-40 (2011).

25. Lipsy, R. J. The national cholesterol education program adult treatment panel III guidelines. J. Manag. Care Pharm. 9, 2-5 (2003).

26. Erejuwa, O. O., Sulaiman, S. A. \& Ab-Wahab, M. S. Modulation of gut microbiota in the management of metabolic disorders: The prospects and challenges. Int. J. Mol. Sci. 15, 4158-4188 (2014).

27. Zhao, W., Liu, Y., Kwok, L. Y., Cai, T. \& Zhang, W. The immune regulatory role of Lactobacillus acidophilus: An updated metaanalysis of randomized controlled trials. Food Biosci. 36, 100656 (2020).

28. Jung, U. J. \& Choi, M. S. Obesity and its metabolic complications: The role of adipokines and the relationship between obesity, inflammation, insulin resistance, dyslipidemia and nonalcoholic fatty liver disease. Int. J. Mol. Sci. 15, 184-223 (2014).

29. Kurutas, E. B. The importance of antioxidants which play the role in cellular response against oxidative/nitrosative stress: Current state. Nutr. J. 15, 71 (2016)

30. Kelly-Quagliana, K. A., Nelson, P. D. \& Buddington, R. K. Dietary oligofructose and inulin modulate immune functions in mice. Nutr. Res. 23, 257-267 (2003).

31. Lomax, A. R., Cheung, L. V. Y., Noakes, P. S., Miles, E. A. \& Calder, P. C. Inulin-type $\beta 2-1$ fructans have some effect on the antibody response to seasonal influenza vaccination in healthy middle-aged humans. Front. Immunol. 6, 490 (2015).

32. Sefidgari-Abrasi, S. et al. Lactobacillus plantarum and inulin: Therapeutic agents to enhance cardiac ob receptor expression and suppress cardiac apoptosis in type 2 diabetic rats. J. Diabetes Res. 2020, 4745389 (2020).

33. Myhill, L. J. et al. Effects of the dietary fibre inulin and Trichuris suis products on inflammatory responses in lipopolysaccharidestimulated macrophages. Mol. Immunol. 121, 127-135 (2020).

34. Anadón, A., Martínez-Larrañaga, M.R., Ares, I. \& Martínez, M.A. Pebiotics and probiotics: An assessment of their safety and health benefits. in Bioactive Foods in Promoting Health: Probiotics and Prebiotics (ed. Watso, R. R. \& Preedy, V.R.) 3-23 (Academic Press, 2016).

35. Jena, P. K. et al. Isolation and characterization of probiotic properties of Lactobacilli isolated from rat fecal microbiota. Microbiol. Immunol. 57, 407-416 (2013).

36. Kanehisa, M. \& Goto, S.KEGG: Kyoto Encyclopedia of Genes and Genomes. Nucleic Acids Res. 28, 27-30 (2000).

37. Kanehisa, M. Toward understanding the origin and evolution of cellular organisms. Protein Sci. 28, 1947-1951 (2019).

\section{Acknowledgements}

This project was funded by the National Research Council of Thailand (NRCT) and supported Mahidol University. We thank Mark Cleasby, PhD, from Edanz Group (https://en-author-services.edanzgroup.com/ac) for editing a draft of this manuscript. Permission from Kanehisa Laboratories is granted to Scientific Reports of Springer Nature Ltd to publish both in print and digital under the CC BY 4.0 open access license the following KEGG pathway map images-MAPK signaling pathway (map04010) — fatty acid elongation (map00062) — glutathione metabolism (map00480).

\section{Author contributions}

W.P. initially the idea for the study. P.P. performed, obtained analyzed and interpreted the experiment in animals data. P.P. was aware of the animal group allocation at the different stages of the experiment. N.K. provide the idea and plan for Lactobacillus identification. T.T. assist to perform the hepatic proteome. O.R. performed and obtained analyzed and interpreted the proteomic data. O.R. and P.P. write a manuscript. All authors read and approved the final manuscript.

\section{Competing interests}

The authors declare no competing interests.

\section{Additional information}

Supplementary Information The online version contains supplementary material available at https://doi. org/10.1038/s41598-021-85427-8.

Correspondence and requests for materials should be addressed to P.P.

Reprints and permissions information is available at www.nature.com/reprints.

Publisher's note Springer Nature remains neutral with regard to jurisdictional claims in published maps and institutional affiliations.

(c) (i)

Open Access This article is licensed under a Creative Commons Attribution 4.0 International License, which permits use, sharing, adaptation, distribution and reproduction in any medium or format, as long as you give appropriate credit to the original author(s) and the source, provide a link to the Creative Commons licence, and indicate if changes were made. The images or other third party material in this article are included in the article's Creative Commons licence, unless indicated otherwise in a credit line to the material. If material is not included in the article's Creative Commons licence and your intended use is not permitted by statutory regulation or exceeds the permitted use, you will need to obtain permission directly from the copyright holder. To view a copy of this licence, visit http://creativecommons.org/licenses/by/4.0/.

(c) The Author(s) 2021 